\title{
Role of Mannheim Peritonitis Index in Perforative Peritonitis in Rural Population
}

\author{
Authors
}

\author{
Venkateswara Rao Katta ${ }^{1}$, Amarendra Prasad N ${ }^{2}$, Ashok Kumar V ${ }^{3}$ \\ ${ }^{1,2,3}$ MNR Medical College and Hospital, Sangareddy, Telangana, India \\ Email: ${ }^{1}$ hasiven@gmail.com, ${ }^{2}$ amarendra_199@yahoo.com, ${ }^{3}$ vashokk11@gmail.com
}

\begin{abstract}
Perforative Peritonitis still remains a disease with higher mortality and morbidity rates in rural population of India. The aim of this study was to evaluate the efficacy of Mannheim Prognostic Index in predicting the clinical outcomes in patients with Perforative Peritonitis.

Material and Methods: Prospective cohort study carried out over a period of 2 years on patients presenting with Perforative peritonitis. MPI score was calculated on admission for all patients and they were followed up till discharge or death and results were analysed.

Results: A total of 100 patients were analysed with a mortality rate of $17 \%$. MPI score $\geq 30$ was significantly associated with Mortality, while a score of 21-29 was associated with $15.20 \%$ mortality. Age $\geq$ 50 yrs, female sex, organ failure, duration of symptoms $\geq 24$ hrs pre operatively, origin of sepsis from colorectal perforations, fecal and purulent nature of peritonitis were found to be independent risk factors for statistically significant risk of post operative complications.

Conclusion: MPI is a valid and reliable predictor for pos operative mortality and morbidity in patients with perforative peritonitis.

Keywords- MPI- Mannheim Peritonitis Index, MOFS- Multi Organ Dysfunction Syndrome, APACHEAcute Physiology And Chronic Health Evaluation.
\end{abstract}

\section{Introduction}

Peritonitis from perforation of abdominal viscera or perforative peritonitis is an acute pathology frequently observed in surgical practice. Perforative peritonitis represents a complex condition due to various etiologies with different prognoses and is associated with an incidence of multiple organ dysfunction syndrome (MOFS) up to $73 \%$ and mortality rates reaching $30 \%$. $^{[1,2]}$

Despite the timely surgical intervention, sophisticated intensive care units, advances in the use of higher dose, last generation antibiotics and a better understanding of pathophysiology, the mortality rate of perforation peritonitis are still high. The outcome of an abdominal infection depends on the complex interaction of many different factors and the success obtained with the early institution of specific therapeutic procedures. It also depends upon the exact recognition of the seriousness of the diseases and an accurate assessment and classification of the patients' risks. Early prognostic evaluation of peritonitis to predict high risk patients who need more aggressive therapeutic procedure such as radical debridement, lavage system, open management and planned relook laparotomy. An accurate risk index classification is the only way to 
settle a standard of comparison between group of patients and different treatment methods which would allow prospective adequate comparative studies. ${ }^{[3]}$

Studies have shown that, a majority of the patients with Perforative peritonitis present late, often with signs of septicemia. ${ }^{[4]}$ These patients are at an increased risk of peri operative morbidity and mortality further complicating the task of the care givers to provide optimal peri operative care. Early prognostic evaluation of abdominal sepsis is desirable to select high risk patients for more aggressive therapeutic procedures and to provide objective classification of the severity of the disease, as also to choose the optimal peri operative anesthetic management strategies. ${ }^{[5]}$

Several scores have been used to identify those risk factors predictive of perforative peritonitis mortality, but most of them are complex to calculate and difficult to use outside intensive care units. Mannheim Peritonitis Index was developed by Wacha and Linder in 1983. It was developed based on the retrospective analysis of data from 1253 patients with peritonitis in which 20 possible risk factors were considered. Of these 20 factors, only 8 were proved to be of prognostic relevance and were entered into Mannheim Peritonitis Index (Table 1). These factors were classified according to their predictive power. Maximal possible score is 47 and minimal possible score is zero. a total score value higher than 26 identified patients at risk of death from severe peritonitis with a good specificity (79\%), sensitivity (84\%) and overall accuracy $(81 \%){ }^{[6]}$

Table 1 Mannheim Peritonitis Index

\begin{tabular}{|c|c|}
\hline Risk Factor & Score \\
\hline Age $>50$ years old & 5 \\
\hline Female sex & 5 \\
\hline Organ failure* & 7 \\
\hline Malignancy & 4 \\
\hline $\begin{array}{l}\text { Pre operative duration } \\
\text { of peritonitis }>24 \mathrm{~h}\end{array}$ & 4 \\
\hline $\begin{array}{l}\text { Origin of sepsis } \\
\text { not colonic }\end{array}$ & 4 \\
\hline
\end{tabular}

Diffuse generalized

peritonitis Exudate

Clear

Cloudy

Fecal

0

6

12

*Organ Failure: Kidney failure: Creatinine level $>177 \mathrm{mmol} / \mathrm{l}$, urea level $>167 \mathrm{mmol} / \mathrm{l}$, oliguria $<20 \mathrm{ml} / \mathrm{h}$; pulmonary insufficiency: PO2 <50 $\mathrm{mmHg}, \mathrm{PCO} 2>50 \mathrm{mmHg}$; shock hypodynamic or hyperdynamic; intestinal obstruction/paralysis or complete mechanical ileus. ${ }^{6}$

Other scores have been proposed for evaluation of patients affected by peritonitis. The acute physiology and chronic health evaluation II score (APACHE II) is widely used in emergency patients and considers many different physiological and clinical parameters. A good correlation of this score with mortality in perforative peritonitis is described in literature, even if it does not include indicators such as type of peritonitis and causes of perforation. ${ }^{[2,7]}$ Nonetheless, it is a complex score which can only be calculated after $24 \mathrm{~h}$ in intensive care unit, while the MPI has showed equivalent efficacy in similar series of patients. ${ }^{[8,9]}$

\section{Material and Methods:}

The study was designed as a prospective study cohort study on a population of patients presenting with perforative peritonitis to MNR Medical Hospital, a tertiary hospital located in rural southern India. Regional ethics committee approval was obtained prior to initiation of study. The study was conducted between 2013 to 2015, with a target to include a total of 100 patients in the study. Only those patients with primary hollow viscous perforation were included in the study. Peritonitis due to anastomotic leak, or perforation following a recent abdominal surgery were excluded from the study.

After an informed consent was obtained from the patients, a thorough history and clinical examination was carried out on all patients. Due attention was given to record the chronology of symptoms till presentation to the hospital. Particular care was taken in recording co-morbidities. Cardiovascular 
abnormalities included, hypertension, ECG changes on presentation, any other condition for which patient has been cardiac medications. Respiratory disease was assessed on chest $X$ ray and any condition for which patient is taking medications. Other co- morbid conditions that were assessed included Diabetes mellitus, hepatic diseases, cerebro vascular diseases and chronic kidney disease were assessed by patient history and as pathologies for which patient was on medication.

At the time of admission, physical findings were recorded meticulously, which included HR, BP, $\mathrm{SpO}_{2}$ and Temperature. All patients underwent erect abdominal and chest X-ray along with ultrasonography of abdomen as initial screening test to confirm the diagnosis of hollow viscous perforation. Routine laboratory investigations were done as part of surgical profile including electrocardiogram and echocardiogram when indicated. After confirmation of diagnosis, all patients had Nasogastric tube insertion, indwelling Foley catheter was inserted and a decision was made for laparotomy for all patients included in the study.

During surgery, the site of perforation was identified and recorded in all cases; the decisions on the surgical procedure and on the need for a stoma were based on intra-operative findings. The site and size of perforation, type of peritonitis (localized or generalized), contamination (serous, purulent, and fecal) and the state of the rest of the gastrointestinal tract and solid organs was noted. An extensive lavage of the abdominal cavity was carried out and depending on the site of perforation one or two drains were positioned in pelvis and hepatorenal pouch of Morrison, at the end of the procedure in all cases.

The data was analyzed for correlations between mortality and clinical factors which were conducted by univariate analysis; their statistical significance was evaluated by means of chi-square test for categorical variables and by means of Student t-test for continuous variables. In order to identify those factors independently related to an increased risk of mortality, a logistic regression model was built. For statistical comparison, a p-value $<0.05$ was considered significant.

\section{Results}

Among the total 100 patients included in the study, 76 patients were male and 24 were female, with a male to female ratio of $3.17: 1$. The mean age of patients in our study was $39.76 \pm 17.37 \mathrm{yrs}$. Total of 55,33 and 12 patients belonged to MPI categories 1 , 2 and $3.65 \%$ of patients presented with in $24 \mathrm{hrs}$ of onset of symptoms. Among all the patients, only $10 \%$ of patients presented with localized peritonitis, while the rest of them had diffuse peritonitis at the time of presentation.

Duodenal or Gastric Perforation was the most common site of hollow viscous perforation seen in $54 \%$ of patients, Ileal or Ileocaecal perforation was the next commonest seen in $29 \%$ of patients, $9 \%$ of patients had colorectal perforation, while appendicular perforation was seen in 5\% and Jejunal perforation accounted for $3 \%$ of cases.

Organ failure was evident in $24 \%$ of patients, with 9 patients $(37.5 \%)$ having only one organ system involvement. 13 patients $(54.17 \%)$ had a combination of 2 organ system failure, while 2 patients $(8.33 \%)$ had combination of 3 organ system failure. Distribution of organ failure is shown in Table 2. Of the 24 patients, that had organ failure, 17 patients $(70.80 \%)$ expired while 7 survived (29.20\%), p-value was significant at $<0.00001$.

Table 2 Correlation of organ failure and clinical outcome

\begin{tabular}{|l|l|l|l|}
\hline Organ Failure & Survived & Expired & Total \\
\hline Renal & $1(16.66 \%)$ & $5(83.34 \%)$ & $6(25 \%)$ \\
\hline Cardiovascular & $1(50 \%)$ & $1(50 \%)$ & $2(8.33 \%)$ \\
\hline Pulmonary & $1(100 \%)$ & 0 & $1(4.17 \%)$ \\
\hline Renal, Cardiovascular & $4(33.3 \%)$ & $8(67.7 \%)$ & $12(50 \%)$ \\
\hline Renal, Pulmonary & 0 & $1(100 \%)$ & $1(4.17 \%)$ \\
\hline $\begin{array}{l}\text { Renal, Pulmonary, } \\
\text { Cardiovascular }\end{array}$ & 0 & $2(100 \%)$ & $2(8.33 \%)$ \\
\hline \begin{tabular}{l} 
Total \\
\hline
\end{tabular} & $7(29.17 \%)$ & $17(70.83 \%)$ & $24(100 \%)$ \\
\hline
\end{tabular}

A univariate analysis for risk of mortality was carried out and is shown in Table 3. 
In our study one patient had malignant perforation arising from distal sigmoid malignancy, which resulted in mortality. 14 out of 50 patients $(28 \%)$, operated $>24 \mathrm{hrs}$ after onset of symptoms have died. Of the 92 patients with diffuse peritonitis, 17 patients $(18.48 \%)$ died.

A total of $43 \%$ of patients developed post operative complications of which, wound infection remained the most common post operative complication seen in 16 patients $(37.21 \%) .3$ patients $(6.98 \%)$ had all three system namely, pulmonary, cardiovascular and renal complications in post operative period, all of whom died. $6(13.95 \%)$ patients had 2out of three organ system complications, while 7 (16.28\%) patients had one of the three system complications. Anastomotic leak with wound infection was seen in $2(4.65 \%)$ of the patients. 1 patient each $(2.32 \%)$ of patients each had paralytic ileus, pulmonary complication with leak, Leak with cardiovascular and renal complications, anastomotic leak and wound infection with cardiovascular complications in the post operative period.

Table 3 Factors related to mortality on univariate analysis

\begin{tabular}{|c|c|c|c|c|}
\hline & & number & Mortality & $\mathrm{p}$-value \\
\hline Age & $\begin{array}{l}<50 \mathrm{yrs} \\
>50 \mathrm{yrs}\end{array}$ & $\begin{array}{l}77 \\
23\end{array}$ & $\begin{array}{l}7(9.09 \%) \\
10(43.48 \%)\end{array}$ & 0.000117 \\
\hline Female Sex & & 24 & $5(20.83 \%)$ & 0.566 \\
\hline Organ Failure & & 24 & $17(70.83 \%)$ & $<0.00001$ \\
\hline Malignancy & & 1 & $1(100 \%)$ & 0.026 \\
\hline \multirow{2}{*}{$\begin{array}{l}\text { Timing } \\
\text { Surgery }\end{array}$} & $<24 \mathrm{hrs}$ & 50 & $3(6 \%)$ & 0.003 \\
\hline & $>24 \mathrm{hrs}$ & 50 & $14(28 \%)$ & \\
\hline \multirow{2}{*}{$\begin{array}{l}\text { Source } \\
\text { sepsis }\end{array}$} & Duodenal/Gastric & 54 & $4(7.41 \%)$ & 0.00011 \\
\hline & $\begin{array}{l}\text { Ileal/Ileocaecal } \\
\text { Junction } \\
\text { Colorectal } \\
\text { Appendicular } \\
\text { Jejunal }\end{array}$ & $\begin{array}{l}29 \\
9 \\
5 \\
3\end{array}$ & $\begin{array}{l}5(17.24 \%) \\
6(66.67 \%) \\
0(0.0 \%) \\
1(33.33 \%)\end{array}$ & \\
\hline \multirow{2}{*}{$\begin{array}{l}\text { Type of } \\
\text { Peritonitis }\end{array}$} & Diffuse & 92 & $17(18.48 \%)$ & 0.182 \\
\hline & Localised & 8 & $0(0.0 \%)$ & \\
\hline \multirow{2}{*}{$\begin{array}{l}\text { Type } \\
\text { Exudate }\end{array}$} & Purulent & 83 & $10(12.04 \%)$ & 0.00001 \\
\hline & $\begin{array}{l}\text { Fecal } \\
\text { Clear }\end{array}$ & $\begin{array}{l}10 \\
7\end{array}$ & $\begin{array}{l}7(70 \%) \\
0(0.0 \%)\end{array}$ & \\
\hline MPI Score & $<21$ & 55 & $0(0.0 \%)$ & 0.00001 \\
\hline
\end{tabular}

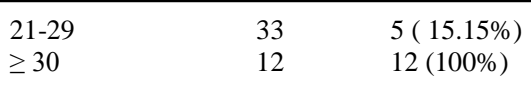

Association between MPI score and complications was also analyzed and found that $11(20 \%)$ out of 44 patients in MPI score group less than 21 had post operative complications. Among the patients with MPI score 21 to 29, 21 (63.6\%) of patients had post operative complications. Of the 12 patients with MPI score $\geq 30,11$ patients $(91.7 \%)$ had complications $(\mathrm{p}<0.00001)$

\section{Discussion}

The prognosis of perforative peritonitis still remains poor despite advances in surgical techniques, antibiotic use and aggressive early interventions in critical care. The mortality rate has been reported between $19 \%$ and 26\%.[10,11] This, predominantly is due to early onset of sepsis, or due to delayed presentation in developing countries like India. The mortality rate in our study was $17 \%$ in keeping with the trends observed elsewhere.

The clinical outcome rates in perforative peritonitis are a direct result of interaction among multiple variables. These factors include pathogenesis of peritonitis, clinical grade of peritonitis, delay in treatment, co morbid conditions of the patents. The last factor in particular, plays a critical role, due to the fact that advances in medical field have prolonged the life expectancy.

Our study has proved the value of MPI score as a powerful predictor of survival for perforative peritonitis. Further subset evaluation of multiple variables has revealed statistically significant increased rates of mortality in patients aged $\geq 50 \mathrm{yrs}$ (p-0.00011). There was significant association between the number of organs failing and the clinical outcomes in our study, with higher the number of organs involved, higher the mortality rates. $100 \%$ mortality was seen when all three organs cardiovascular, pulmonary and renal systems failed, while $88.3 \%$ mortality was noted with renal failure alone and $66.7 \%$ mortality observed when combination of renal and cardiovascular systems failed ( $\mathrm{p}<0.00001)$. 
Only one patient presented with recto-sigmoid perforation, who subsequently expired and there was a significant correlation between malignancy and mortality ( $\mathrm{p} 0.026)$. Highest rates of mortality were seen in colorectal perforation $(66.7 \%$, p0.00011).

Post operative complication rates were $43 \%$, of whom 15 patients expired with a mortality rate of $34.9 \%$ (p-3.55E-05), which was clinically significant. All 12 patients with MPI score $\geq 30$ have died, while $15.2 \%$ (5 out of 33 patients), with a score of 21-29 died. There were no recorded deaths in patients with MPI score $<21$. This association was found to be significant with a $p$ value $6.52 \mathrm{E}-16$ on Pearson Chi-Square test. Seventeen patients who died in our study had an MPI score 32.06 \pm 4.31 , while those who survived had MPI of $19.11 \pm 4.79$, the difference between the groups was statistically significant on unpaired $t$ test.

MPI Score has been validated in several studies, and has been reported to be an effective and a reliable tool at identifying the subsets of patients with perforative peritonitis who carry a higher risk of mortality, although there were discrepancies among the cut off values. $[5,12,13]$ With a cut off value at 26 our study showed a sensitivity of $94.1 \%$ and a specificity of $94 \%$.

Post operative complication rates were significantly higher $(\mathrm{p}<0.00001)$ in sub group of patients with MPI $\geq 30$, seen in $91.7 \%$ of patients, whereas $63.6 \%$ patients with a score of 21-29 had post operative complications. The complication rates were lowest in MPI score <21 seen in only $20 \%$ of patients. Further subset analysis found age $\geq 50 \mathrm{yrs}$, female sex, organ failure, duration of symptoms $\geq 24 \mathrm{hrs}$ pre operatively, origin of sepsis from colorectal perforations, fecal and purulent nature of peritonitis were found to be independent risk factors for statistically significant risk of post operative complications.

\section{Conclusion}

Based on the results from our study, we conclude that Mannheim Peritonitis Index is a reliable tool in predicting clinical outcomes in patients with perforative peritonitis. MPI score $\geq 30$ was strongly associated with poor outcomes both in terms of mortality and post operative complications. Early objective identification of such high risk patients would enable the care givers to adopt a more specific goal directed therapy and may help in reducing the mortality and morbidity rates in patients with perforative peritonitis.

\section{Acknowledgements}

We would like to thank Prof Ashok Kumar V, who was instrumental in conceptualizing this study and has critically reviewed this article. Our sincere thanks would also be towards the department of Preventive and Social medicine, MNR medical College and Hospital for their assistance with statistical analysis of the data presented.

Conflict of Interest; None declared.

\section{References}

1. P.S. Barie, L.J. Hydo, E. Fisher, Development of multiple organ dysfunction syndrome in critically ill patients with perforated viscous. Predictive value of APACHE severity scoring, Arch. Surg. 131 (1996) 37-43.

2. J.M. Bohnen, R.A. Mustard, S.E. Oxholm, B.D. Schouten, APACHE II score and abdominal sepsis. A prospective study, Arch. Surg. 123 (1988) 225-229.

3. Tushar Dani, Prof. L. Ramachandra, Rajesh Nair, Digvijoy Sharma. Evaluation of Prognosis in patients' with perforation peritonitis using Mannheim's Peritonitis Index. International Journal of Scientific and Research Publications, Volume 5, Issue 5, May 2015.

4. Ersumo T, W/Meskel Y, Kotisso B. Perforated peptic ulcer in Tikur Anbessa Hospital; a review of 74 cases. Ethiop Med J 2005;43:913.

5. Billing A, Fröhlich D, Schildberg FW. Prediction of outcome using the Mannheim 
peritonitis index in 2003 patients. Br J Surg 1994;81:20913.

6. Linder MM, Wacha H. The Mannheim peritonitis index. An instrument for the intra operative prognosis of peritonitis. Chirurg,1987, Feb ; 58 (2) 84-92.

7. S.V. Kulkarni, A.S. Naik, N. Subramanian, APACHE-II scoring system in perforative peritonitis, Am. J. Surg. 94 (2007) 549-552.

8. F. Pacelli, G.B. Doglietto, S. Alfieri, E. Piccioni, A. Sgadari, D. Gui, F. Crucitti, Prognosis in intra-abdominal infections. Multivariate analysis on 604 patients, Arch. Surg. 131 (1996) 641-645.

9. K. Bosscha, K. Reijnders, P.F. Hulstaert, A. Algra, C. van der Werken, Prognostic scoring systems to predict outcome in peritonitis and intra-abdominal sepsis, Br. J. Surg. 84 (1997) 1532-1534.

10. S. Biondo, E. Ramos, D. Fraccalvieri, E. Kreisler, J.M. Ragu_e, E. Jaurrieta, Comparative study of left colonic peritonitis severity score and Mannheim peritonitis index, Br. J. Surg. 93 (2006) 616-622.

11. K.K. Tan, S.L. Bang, R. Sim, Surgery for small bowel perforation in an Asian population: predictors of morbidity and mortality, J. Gastrointest. Surg. 14 (2010) 493-499.

12. H. Sawayama, S. Tomiyasu, K. Kanemitsu, T. Matsumoto, H. Tanaka, H. Baba, Colonic perforation due to colorectal cancer: predicting postoperative organ failure with a preoperative scoring system and selecting the optimal surgical method based on the prognosis, Surg. Today 42 (2012) 10821087.

13. J.T. Mäkelä, H. Kiviniemi, S. Laitinen, Prognostic factors of perforated sigmoid diverticulitis in the elderly, Dig. Surg. 22 (2005) 100-106. 